\title{
Novel Stabilization Conditions for Uncertain Singular Systems with Time-Varying Delay
}

\author{
Yuanchun Ding, ${ }^{1}$ Falu Weng, ${ }^{2}$ Ji Ge, ${ }^{3}$ Qi Liang, ${ }^{1}$ and Xueyi Zhi ${ }^{1}$ \\ ${ }^{1}$ Jiangxi Key Laboratory of Mining Engineering, Jiangxi University of Science and Technology, Ganzhou, Jiangxi 341000, China \\ ${ }^{2}$ Faculty of Electrical Engineering and Automation, Jiangxi University of Science and Technology, Ganzhou, Jiangxi 341000, China \\ ${ }^{3}$ Department of Mechanical and Industrial Engineering, University of Toronto, Toronto, ON, Canada M5S $3 G 8$
}

Correspondence should be addressed to Falu Weng; wengfalu@hotmail.com

Received 31 March 2014; Accepted 11 June 2014; Published 22 June 2014

Academic Editor: Zhengguang Wu

Copyright (C) 2014 Yuanchun Ding et al. This is an open access article distributed under the Creative Commons Attribution License, which permits unrestricted use, distribution, and reproduction in any medium, provided the original work is properly cited.

\begin{abstract}
The problem of delay-dependent robust stabilization for continuously singular time-varying delay systems with norm-bounded uncertainties is investigated in this paper. First, based on some mathematical transform, the uncertain singular system is described in a form which involves the time-delay integral items. Then, in terms of the delay-range-dependent Lyapunov functional and the LMI technique, the improved delay-dependent LMIs-based conditions are established for the uncertain singular systems with time-varying delay to be regular, causal, and stable. Furthermore, by solving these LMIs, an explicit expression for the desired state feedback control law can be obtained; thus, the regularity, causality, and stability of the closed-loop system are guaranteed. In the end, numerical examples are given to illustrate the effectiveness of the proposed methods.
\end{abstract}

\section{Introduction}

During the past several decades, singular systems, which are known as descriptor systems, implicit systems, and differential-algebraic systems, received a considerable attention because of their applications in many areas, such as engineering systems, social systems, economic systems, network analysis, biological systems, and time-series analysis $[1,2]$. As we all know, it is required to consider not only stability but also regularity and absence of impulses (for continuous singular systems) or causality (for discrete singular systems) simultaneously for singular systems; thus, the study on singular systems is much more complicated than that on the regular ones $[3,4]$. Recently, many scholars have applied themselves to the research of singular system and many stability and stabilization conditions have been established for singular systems; see, for example, [5-11] and the references therein.

On the other hand, much attention has been paid to the study of time-delay systems in recent years, because time delays inevitably exist in a variety of practical systems, such as chemical processes, nuclear reactors, and biological systems, and lead to the instability and poor performance of systems
[12-15]. Generally speaking, the existing results can be classified into two types: delay-independent results (see, e.g., [16, 17] and the references therein) and delay-dependent results (see, e.g., $[18,19]$ and the references therein). Furthermore, the delay-independent case is regarded as more conservative than the delay-dependent case, especially when the time delay is comparatively small. Thus, the delay-dependent stability and stabilization conditions for singular time-delay systems have received increasing attention during the past years. For example, by utilizing model transformation and bounding technique for cross-terms, Zhu et al. [20, 21] investigated the delay-dependent robust stabilization problem for uncertain singular time-delay systems. References [22, 23] also discussed the problem, and neither model transformation nor bounding technique for cross-terms is needed in the development of the results. Based on an improved Lyapunov functional, which includes some nonpositive items, Weng and Mao [24] discussed the delay-dependent robust stability and stabilization for uncertain singular time-delay systems, and some LMIs-based results were obtained. However, in the practical systems, most of those delays are time-varying because the external perturbances and uncertainties are 
always existing [25]. Thus, proposing some time-varying delay-tolerant results for the singular system is obviously more meaningful. In terms of Lyapunov stability theory and LMI technique, some results about the admissibility and dissipativity for discrete-time singular systems with mixed time-varying delays were proposed in [26]. Based on the probability idea, Weng and Mao [27] presented some delay-range-dependent and delay-distribution-independent stability criteria for discrete-time singular systems with timevarying delay, and several sufficient results were obtained. However, to the best of our knowledge, the stabilization conditions for singular time-varying delay systems still have not been fully investigated, and there is still much room for improvement.

This paper is concerned with the problem of robust stabilization for continuously singular time-varying delay systems with norm-bounded and time-varying parametric uncertainties. The focus of this paper is to design a state feedback controller such that the closed-loop system is regular, causal, and stable for all admissible uncertainties. The proposed sufficient robust stabilization conditions of the considered system are described in terms of strict LMIs, which are formulated in terms of all the coefficient matrices of the original system. Finally, numerical examples are provided to demonstrate the effectiveness of the proposed methods.

Notation 1. Throughout this paper, for real matrices $X$ and $Y$, the notation $X \geq Y$ (resp., $X>Y$ ) means that the matrix $X-Y$ is semipositive definite (resp., positive definite). $I$ is the identity matrix with appropriate dimensions; a superscript " $T$ " represents transpose. $\|x\|$ refers to the Euclidean norm of the vector $x$. For a symmetric matrix, $*$ denotes the symmetric terms. $M+M^{T}$ is denoted as $\{M\}^{H}$ for simplicity.

\section{Problem Formulation and Dynamic Models}

Consider an uncertain singular system with time-varying delay described by Wu and Zhou [28]:

$$
\begin{array}{r}
E \dot{x}_{(t)}=(A+\Delta A) x_{(t)}+\left(A_{d}+\Delta A_{d}\right) x_{(t-d(t))}+B u(t), \\
x_{(t)}=\Phi_{(t)}, \quad t \in\left[-d_{2}, 0\right],
\end{array}
$$

where $x_{(t)} \in R^{n}$ is the state variable and $d_{(t)}$ is a time-varying delay satisfying $0<d_{1} \leq d_{(t)} \leq d_{2}$ and $\dot{d}_{(t)} \leq \mu$. $\Phi_{(t)}$ is a compatible initial value at $t$. The matrix $E \in R^{n \times n}$ may be singular and rank $E \leq n$ is assumed. $A, A_{d}$, and $B$ are real constant matrices with appropriate dimensions. $\Delta A$ and $\Delta A_{d}$ are norm-bounded parametric matrices and are assumed to be of the following form:

$$
\left[\begin{array}{ll}
\Delta A & \Delta A_{d}
\end{array}\right]=M F_{(t)}\left[\begin{array}{ll}
N_{1} & N_{2}
\end{array}\right]
$$

where $F_{(t)} \in R^{k \times s}$ is an unknown parameter matrix satisfying $F_{(t)}^{T} F_{(t)} \leq I . M, N_{1}$, and $N_{2}$ are known constant matrices with appropriate dimensions.
The nominal unforced singular time-delay system of (1) can be described as

$$
\begin{aligned}
E \dot{x}_{(t)} & =A x_{(t)}+A_{d} x_{\left(t-d_{(t)}\right)}, \\
x_{(t)} & =\Phi_{(t)}, \quad t \in\left[-d_{2}, 0\right] .
\end{aligned}
$$

The following definitions and lemmas will be used in the proof of the main results.

Definition 1 (see [29]). (i) The pair $(E, A)$ is regular if $\operatorname{det}(S E-A)$ is not identically zero; (ii) the pair $(E, A)$ is said to be impulse free if it is regular and $\operatorname{deg}\{\operatorname{det}(S E-A)\}=$ rank $E$.

Definition 2 (see [29]). (i) The system (3) is said to be regular and impulse free if $d_{(t)}$ satisfies $0<d_{1} \leq d_{(t)} \leq d_{2}$; the pair $(E, A)$ is regular and impulse free. (ii) The system (3) is said to be stable if, for any $\varepsilon>0$, there exists a scalar $\mu(\varepsilon)$, such

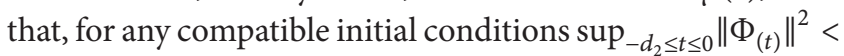
$\mu(\varepsilon)$, when $t>0$, the solution $x(t)$ of the system (3) satisfies $\|x(t)\| \leq \varepsilon$. Furthermore, $\lim _{t \rightarrow 0} x(t)=0$. (iii) The system (3) is said to be admissible if it is regular, impulse free, and stable.

Definition 3. (i) The uncertain singular time-varying delay system (1) is said to be robustly admissible if the system (1) with $u(t)=0$ is regular, impulse free, and stable for all admissible uncertainties satisfying (2) and any time-delay $d_{(t)}$ satisfying $0<d_{1} \leq d_{(t)} \leq d_{2}$. (ii) The singular timevarying delay system (1) is said to be stabilizable if there exists state feedback controller $u(t)=K x(t)$ such that the closedloop system is admissible for any time delay $d_{(t)}$ satisfying $0<d_{1} \leq d_{(t)} \leq d_{2}$. (iii) The uncertain singular time-varying delay system (1) is said to be robustly stabilizable if there exists state feedback controller $u(t)=K x(t)$ such that the closed-loop system is robustly admissible for all admissible uncertainties satisfying (2) and any time-delay $d_{(t)}$ satisfying $0<d_{1} \leq d_{(t)} \leq d_{2}$.

After some mathematical transform, the systems (1) and (3) can be described in the following forms:

$$
\begin{aligned}
E \dot{x}_{(t)}= & \left(A+\Delta A+A_{d}+\Delta A_{d}\right) x_{(t)} \\
& -\left(A_{d}+\Delta A_{d}\right) \sum_{i=0}^{m-1} \int_{t-((i+1) / m) d_{1}}^{t-(i / m) d_{1}} \dot{x}_{(s)} d s \\
& -\left(A_{d}+\Delta A_{d}\right) \int_{t-d(t)}^{t-d_{1}} \dot{x}_{(s)} d s, \\
x_{(t)}=\Phi_{(t)}, t \in\left[-d_{2}, 0\right] & \\
E \dot{x}_{(t)}= & \left(A+A_{d}\right) x_{(t)}-A_{d} \sum_{i=0}^{m-1} \int_{t-((i+1) / m) d_{1}}^{t-(i / m) d_{1}} \dot{x}_{(s)} d s
\end{aligned}
$$




$$
\begin{aligned}
-A_{d} \int_{t-d(t)}^{t-d_{1}} \dot{x}_{(s)} d s & \\
& x_{(t)}=\Phi_{(t)}, \quad t \in\left[-d_{2}, 0\right] .
\end{aligned}
$$

For description in brevity, we define $\gamma=d_{1} / m$ and $d_{12}=d_{2}-$ $d_{1}$. This section is concluded by presenting a lemma, which will be used in the proof of our main results.

Lemma 4 (see $[30,31]$ ). Given matrices $\chi, \mu$, and $v$ with appropriate dimensions and with $\chi$ symmetrical, then $\chi+$ $\mu F_{(t)} \nu+\nu^{T} F_{(t)}^{T} \mu^{T}<0$ holds for any $F_{(t)} \in R^{k \times s}$ satisfying $F_{(t)}^{T} F_{(t)} \leq I$, if and only if there exists a scalar $\sigma>0$ such that $\chi+\sigma \mu \mu^{T}+\sigma^{-1} v^{T} v<0$.

\section{Main Results}

In this section, the delay-dependent conditions for system (1) to be stabilizable and robustly stabilizable are presented. As a basis, we first study system (5) and obtain the following Theorem 5.

Theorem 5. For the prescribed scalars satisfying $0<d_{1} \leq d_{2}$, the singular time-delay system (5) is admissible for any timedelay $d_{(t)}$ satisfying $0<d_{1} \leq d_{(t)} \leq d_{2}$ if there exist positive symmetric matrices $P, U_{i}(i=1,2,3,4), Q_{1}, Q_{2}$, matrices $H_{i}(i=1,2), S_{i}(i=1,2,3,4,5,6,7), G_{i}(i=1,2,3,4$, $5,6,7,8)$, and $L_{i}(i=1,2,3,4,5)$ satisfying the following LMIs:

$$
\Psi_{i}=\left[\begin{array}{cccccccc}
\Psi_{111} & \Psi_{112} & G_{1} & -G_{1} & \Psi_{115} & \Psi_{116} & -L_{1}+L_{2}^{T} & L_{5}^{T} \\
* & \Psi_{i 22} & G_{7} & -G_{7} & \Psi_{125} & \Psi_{126} & -L_{2}^{T}-L_{4} & -L_{5}^{T} \\
* & * & \Psi_{133} & \Psi_{134} & \Psi_{135} & \Psi_{136} & G_{2}^{T} & G_{8}^{T} \\
* & * & * & \Psi_{44} & \Psi_{145} & \Psi_{146} & -G_{2}^{T} & -G_{8}^{T} \\
* & * & * & * & \Psi_{155} & \Psi_{156} & L S_{2}^{T}-L_{3} & L S_{7}^{T} \\
* & * & * & * & * & \Psi_{166} & -G_{2}^{T}-L_{2}^{T} & \Psi_{168} \\
* & * & * & * & * & * & \Psi_{i 77} & \Psi_{i 78} \\
* & * & * & * & * & * & * & \Psi_{i 88}
\end{array}\right]<0, \quad i=1,2,
$$

where $L \in R^{n \times(n-r)}$ is any matrix with full columns and satisfies $E^{T} L=0$ and

$$
\begin{gathered}
\Psi_{111}=U_{1}+U_{2}+U_{3}+U_{4}+\left\{L_{1}+H_{1}\left(A+A_{d}\right)\right\}^{H}, \\
\Psi_{112}=-H_{1} A_{d}-L_{1}+L_{4}^{T}, \\
\Psi_{116}=-m H_{1} A_{d}-G_{1}-L_{1}, \\
\Psi_{115}=E^{T} P-H_{1}+\left(H_{2}\left(A+A_{d}\right)\right)^{T}+S_{1} L^{T}+L_{3}^{T}, \\
\Psi_{122}=-3 E^{T} Q_{2} E-L_{4}-L_{4}^{T}, \\
\Psi_{126}=-G_{7}-L_{4}, \\
\Psi_{125}=-\left(H_{2} A_{d}\right)^{T}+S_{6} L^{T}-L_{3}^{T}, \\
\Psi_{133}=-U_{1}+\left\{G_{3}\right\}^{H}, \quad \Psi_{134}=-G_{3}+G_{4}^{T}, \\
\Psi_{135}=S_{3} L^{T}+G_{5}^{T}, \quad \Psi_{136}=-G_{3}+G_{6}^{T}, \\
\Psi_{144}=-U_{2}-\left\{G_{4}\right\}^{H}, \quad \Psi_{145}=S_{4} L^{T}-G_{5}^{T}, \\
\Psi_{146}=-G_{4}-G_{6}^{T}, \quad \Psi_{155}=\gamma^{2} Q_{1}+d_{12}^{2} Q_{2}-\left\{H_{2}\right\}^{H}, \\
\Psi_{156}=-m H_{2} A_{d}+L S_{5}^{T}-G_{5}-L_{3}, \\
\Psi_{166}=-E^{T} Q_{1} E-\left\{G_{6}\right\}^{H}, \\
\Psi_{5}^{T}-L_{5}^{T},
\end{gathered}
$$

$$
\begin{gathered}
\Psi_{178}=-L_{5}^{T}+E^{T} Q_{2} E, \\
\Psi_{177}=-(1-\mu) U_{3}-\left\{L_{2}\right\}^{H}-E^{T} Q_{2} E, \\
\Psi_{188}=-U_{4}-E^{T} Q_{2} E, \\
\Psi_{222}=-E^{T} Q_{2} E-\left\{L_{4}\right\}^{H}, \\
\Psi_{277}=-(1-\mu) U_{3}-\left\{L_{2}\right\}^{H}-3 E^{T} Q_{2} E, \\
\Psi_{278}=-L_{5}^{T}+3 E^{T} Q_{2} E, \\
\Psi_{288}=-U_{4}-3 E^{T} Q_{2} E .
\end{gathered}
$$

Proof. Under the conditions of Theorem 5, it is first shown that the system (5) is regular and impulse free for any timedelay $d_{(t)}$ satisfying $0<d_{1} \leq d_{(t)} \leq d_{2}$. Define $H=$ $\left[\begin{array}{llllllll}I_{n} & I_{n} & 0 & 0 & A^{T} & 0 & 0 & 0\end{array}\right]$. Then, by pre- and postmultiplying (6) by $H$ and $H^{T}$, respectively, it is possible to obtain

$$
\begin{aligned}
& -E^{T} Q_{2} E+A^{T} P E+E^{T} P A+A^{T} L\left(S_{1}+S_{6}\right)^{T} \\
& +\left(S_{1}+S_{6}\right) L^{T} A<0 .
\end{aligned}
$$

Since $\operatorname{rank} E=r \leq n$, there exist two nonsingular matrices $\widetilde{M}$ and $\widetilde{N}$ such that $\widetilde{M} E \widetilde{N}=\left[\begin{array}{ll}I_{r} & 0 \\ 0 & 0\end{array}\right]$. Accordingly, denote $\widetilde{M} A \widetilde{N}=\left[\begin{array}{ll}A_{1} & A_{2} \\ A_{3} & A_{4}\end{array}\right], \widetilde{N}^{T}\left(S_{1}+S_{6}\right)=\left[\begin{array}{l}S_{11} \\ S_{12}\end{array}\right], \widetilde{M}^{-T} L=\left[\begin{array}{l}0 \\ \bar{L}_{1}\end{array}\right]$, where 
$\bar{L}_{1} \in R^{(n-r) \times(n-r)}$ is any nonsingular matrix and $I_{r} \in R^{r \times r}$ is an identity matrix. Then, by pre- and postmultiplying inequalities (8) by $\widetilde{N}^{T}$ and $\widetilde{N}$, respectively, it is possible to obtain

$$
\left[\begin{array}{cc}
\diamond & \diamond \\
* & S_{12} L_{1}^{T} A_{4}+A_{4}^{T} L_{1} S_{12}^{T}
\end{array}\right]<0
$$

Here, " $\diamond$ " representing the matrix blocks are irrelevant to the following discussion; the real expression of these two variables is omitted here. From (9), it is possible to obtain that

$$
S_{12} L_{1}^{T} A_{4}+A_{4}^{T} L_{1} S_{12}^{T}<0 .
$$

It can be shown that $A_{4}$ is nonsingular. Thus, the pair $(E, A)$ is regular and impulse free [29]; that is to say, system (5) is regular and impulse free.

Then, we are in a position to show that system (5) is stable under the conditions of Theorem 5. Choose a LyapunovKrasovskii functional candidate as

$$
V(t)=V_{1}(t)+V_{2}(t)+V_{3}(t),
$$

where

$$
\begin{aligned}
V_{1}(t)= & x_{(t)}^{T} E^{T} P E x_{(t)}+\sum_{i=0}^{m-1} \int_{t-i \gamma}^{t} x_{(s)}^{T} U_{1} x_{(s)} d s \\
& +\sum_{i=0}^{m-1} \int_{t-(i+1) \gamma}^{t} x_{(s)}^{T} U_{2} x_{(s)} d s+\int_{t-d_{t}}^{t} x_{(s)}^{T} U_{3} x_{(s)} d s \\
& +\int_{t-d_{2}}^{t} x_{(s)}^{T} U_{4} x_{(s)} d s, \\
V_{2}(t)= & \gamma \sum_{i=0}^{m-1} \int_{-\gamma}^{0} \int_{t-i \gamma+\varepsilon}^{t-i \gamma} x_{(s)}^{T} Q_{1} x_{(s)} d s d \varepsilon, \\
V_{3}(t)= & d_{12} \int_{-d_{2}}^{-d_{1}} \int_{t+\varepsilon}^{t} \dot{x}_{(s)}^{T} E^{T} Q_{2} E \dot{x}_{(s)} d s d \varepsilon .
\end{aligned}
$$

The derivative of $V(t)$ along the trajectories of (5) satisfies

$$
\dot{V}(t)=\dot{V}_{1}(t)+\dot{V}_{2}(t)+\dot{V}_{3}(t),
$$

where

$$
\begin{aligned}
\dot{V}_{1}(t)= & \dot{x}_{(t)}^{T} E^{T} P E x_{(t)}+m x_{(t)}^{T} U_{1} x_{(t)} \\
& -\sum_{i=0}^{m-1} x_{(t-i \gamma)}^{T} U_{1} x_{(t-i \gamma)}+m x_{(t)}^{T} U_{2} x_{(t)} \\
& -\sum_{i=0}^{m-1} x_{(t-(i+1) \gamma)}^{T} U_{2} x_{(t-(i+1) \gamma)} \\
& +x_{(t)}^{T} U_{3} x_{(t)}-(1-\mu) x_{(t-d(t))}^{T} U_{3} x_{(t-d(t))} \\
& +x_{(t)}^{T} U_{4} x_{(t)}-x_{\left(t-d_{2}\right)}^{T} U_{4} x_{\left(t-d_{2}\right)},
\end{aligned}
$$

$$
\begin{aligned}
\dot{V}_{2}(t) \leq & \gamma^{2} \dot{x}_{(t)}^{T} E^{T} Q_{1} E \dot{x}_{(t)} \\
& -\sum_{i=0}^{m-1} \int_{t-(i+1) \gamma}^{t-i \gamma} \dot{x}_{(s)}^{T} d s E^{T} Q_{1} E \int_{t-(i+1) \gamma}^{t-i \gamma} \dot{x}_{(s)} d s, \\
\dot{V}_{3}(t)= & d_{12}^{2} \dot{x}_{(t)}^{T} E^{T} Q_{2} E \dot{x}_{(t)} \\
& -d_{12} \int_{t-d_{(t)}}^{t-d_{1}} \dot{x}_{(s)}^{T} E^{T} Q_{2} E \dot{x}_{(s)} d s \\
& -d_{12} \int_{t-d_{2}}^{t-d_{(t)}} \dot{x}_{(s)}^{T} E^{T} Q_{2} E \dot{x}_{(s)} d s \\
\leq & d_{12}^{2} \dot{x}_{(t)}^{T} E^{T} Q_{2} E \dot{x}_{(t)} \\
& -\frac{d_{12}}{d_{(t)}-d_{1}} \int_{t-d_{(t)}}^{t-d_{1}} \dot{x}_{(s)}^{T} d s E^{T} Q_{2} E \int_{t-d_{(t)}}^{t-d_{1}} \dot{x}_{(s)} d s \\
& -\frac{d_{12}}{d_{2}-d_{(t)}} \int_{t-d_{2}}^{t-d_{(t)}} \dot{x}_{(s)}^{T} d s E^{T} Q_{2} E \int_{t-d_{2}}^{t-d_{t}} \dot{x}_{(s)} d s .
\end{aligned}
$$

We have $-d_{12} /\left(d_{(t)}-d_{1}\right)-d_{12} /\left(d_{2}-d_{(t)}\right) \leq 4$; thus, when $d_{1} \leq d_{(t)} \leq\left(d_{1}+d_{2}\right) / 2$, it is easy to obtain

$$
\begin{aligned}
& -\frac{d_{12}}{d_{(t)}-d_{1}} \int_{t-d_{(t)}}^{t-d_{1}} \dot{x}_{(s)}^{T} d s E^{T} Q_{2} E \int_{t-d_{(t)}}^{t-d_{1}} \dot{x}_{(s)} d s \\
& \quad \leq\left(-4+\frac{d_{12}}{d_{2}-d_{(t)}}\right) \int_{t-d_{(t)}}^{t-d_{1}} \dot{x}_{(s)}^{T} d s E^{T} Q_{2} E \int_{t-d_{(t)}}^{t-d_{1}} \dot{x}_{(s)} d s .
\end{aligned}
$$

By defining $\theta_{1}(t)=2-d_{12} /\left(d_{2}-d_{(t)}\right)$ and according to $d_{1} \leq$ $d_{(t)} \leq\left(d_{1}+d_{2}\right) / 2$, we have $0 \leq \theta_{1}(t) \leq 1$. Thus, based on the convex theory, it is easy to obtain that

$$
\begin{aligned}
(-4 & \left.+\frac{d_{12}}{d_{2}-d_{(t)}}\right) \int_{t-d_{(t)}}^{t-d_{1}} \dot{x}_{(s)}^{T} d s E^{T} Q_{2} E \int_{t-d_{(t)}}^{t-d_{1}} \dot{x}_{(s)} d s \\
& -\frac{d_{12}}{d_{2}-d_{(t)}} \int_{t-d_{2}}^{t-d_{(t)}} \dot{x}_{(s)}^{T} d s E^{T} Q_{2} E \int_{t-d_{2}}^{t-d_{(t)}} \dot{x}_{(s)} d s \\
= & \left(\frac{1}{2}+\frac{\theta_{1}(t)}{2}\right) \\
& \times\left(-3 \int_{t-d_{(t)}}^{t-d_{1}} \dot{x}_{(s)}^{T} d s E^{T} Q_{2} E \int_{t-d_{(t)}}^{t-d_{1}} \dot{x}_{(s)} d s\right. \\
& \left.-\int_{t-d_{2}}^{t-d_{(t)}} \dot{x}_{(s)}^{T} d s E^{T} Q_{2} E \int_{t-d_{2}}^{t-d_{(t)}} \dot{x}_{(s)} d s\right) \\
& +\left(1-\left(\frac{1}{2}+\frac{\theta_{1}(t)}{2}\right)\right)
\end{aligned}
$$


Mathematical Problems in Engineering

5

$$
\begin{aligned}
\times(- & \int_{t-d_{(t)}}^{t-d_{1}} \dot{x}_{(s)}^{T} d s E^{T} Q_{2} E \int_{t-d_{(t)}}^{t-d_{1}} \dot{x}_{(s)} d s \\
& \left.-3 \int_{t-d_{2}}^{t-d_{(t)}} \dot{x}_{(s)}^{T} d s E^{T} Q_{2} E \int_{t-d_{2}}^{t-d_{(t)}} \dot{x}_{(s)} d s\right) .
\end{aligned}
$$

When $\left(d_{1}+d_{2}\right) / 2 \leq d_{(t)} \leq d_{2}$, we have

$$
\begin{aligned}
& -\frac{d_{12}}{d_{2}-d_{(t)}} \int_{t-d_{2}}^{t-d_{(t)}} \dot{x}_{(s)}^{T} d s E^{T} Q_{2} E \int_{t-d_{2}}^{t-d_{(t)}} \dot{x}_{(s)} d s \\
& \quad \leq\left(-4+\frac{d_{12}}{d_{(t)}-d_{1}}\right) \int_{t-d_{2}}^{t-d_{(t)}} \dot{x}_{(s)}^{T} d s E^{T} Q_{2} E \int_{t-d_{2}}^{t-d_{(t)}} \dot{x}_{(s)} d s .
\end{aligned}
$$

By defining $\theta_{2}(t)=2-d_{12} /\left(d_{(t)}-d_{1}\right)$ and according to $\left(d_{1}+\right.$ $\left.d_{2}\right) / 2 \leq d_{(t)} \leq d_{2}$, we have $0 \leq \theta_{2}(t) \leq 1$. Based on convex theory, we achieve

$$
\begin{aligned}
-\frac{d_{12}}{d_{(t)}-d_{1}} \int_{t-d_{(t)}}^{t-d_{1}} \dot{x}_{(s)}^{T} d s E^{T} Q_{2} E \int_{t-d_{(t)}}^{t-d_{1}} \dot{x}_{(s)} d s \\
+\left(-4+\frac{d_{12}}{d_{(t)}-d_{1}}\right) \int_{t-d_{2}}^{t-d_{(t)}} \dot{x}_{(s)}^{T} d s E^{T} Q_{2} E \int_{t-d_{2}}^{t-d_{(t)}} \dot{x}_{(s)} d s \\
=\left(\frac{1}{2}+\frac{\theta_{2}(t)}{2}\right) \\
\quad \times\left(-\int_{t-d_{(t)}}^{t-d_{1}} \dot{x}_{(s)}^{T} d s E^{T} Q_{2} E \int_{t-d_{(t)}}^{t-d_{1}} \dot{x}_{(s)} d s\right. \\
\left.\quad-3 \int_{t-d_{2}}^{t-d_{(t)}} \dot{x}_{(s)}^{T} d s E^{T} Q_{2} E \int_{t-d_{2}}^{t-d_{(t)}} \dot{x}_{(s)} d s\right) \\
+\left(1-\left(\frac{1}{2}+\frac{\theta_{2}(t)}{2}\right)\right) \\
\times\left(-3 \int_{t-d_{(t)}}^{t-d_{1}} \dot{x}_{(s)}^{T} d s E^{T} Q_{2} E \int_{t-d_{(t)}}^{t-d_{1}} \dot{x}_{(s)} d s\right. \\
\left.\quad-\int_{t-d_{2}}^{t-d_{(t)}} \dot{x}_{(s)}^{T} d s E^{T} Q_{2} E \int_{t-d_{2}}^{t-d_{(t)}} \dot{x}_{(s)} d s\right) .
\end{aligned}
$$

It is easy to obtain $0 \leq\left(1+\theta_{1}(t)\right) / 2 \leq 1$ and $0 \leq(1+$ $\left.\theta_{2}(t)\right) / 2 \leq 1$. Then, based on the analysis mentioned above, we can obtain that when $\theta(t)$ satisfies $0 \leq \theta(t) \leq 1$, there exists

$$
\begin{aligned}
& -\frac{d_{12}}{d_{(t)}-d_{1}} \int_{t-d_{(t)}}^{t-d_{1}} \dot{x}_{(s)}^{T} d s E^{T} Q_{2} E \int_{t-d_{(t)}}^{t-d_{1}} \dot{x}_{(s)} d s \\
& -\frac{d_{12}}{d_{2}-d_{(t)}} \int_{t-d_{2}}^{t-d_{(t)}} \dot{x}_{(s)}^{T} d s E^{T} Q_{2} E \int_{t-d_{2}}^{t-d_{(t)}} \dot{x}_{(s)} d s
\end{aligned}
$$

$$
\begin{array}{r}
\leq \theta(t)\left(-3 \int_{t-d_{(t)}}^{t-d_{1}} \dot{x}_{(s)}^{T} d s E^{T} Q_{2} E \int_{t-d_{(t)}}^{t-d_{1}} \dot{x}_{(s)} d s\right. \\
\left.+\int_{t-d_{2}}^{t-d_{(t)}} \dot{x}_{(s)}^{T} d s E^{T} Q_{2} E \int_{t-d_{2}}^{t-d_{(t)}} \dot{x}_{(s)} d s\right) \\
+(1-\theta(t)) \\
\times\left(-\int_{t-d_{(t)}}^{t-d_{1}} \dot{x}_{(s)}^{T} d s E^{T} Q_{2} E \int_{t-d_{(t)}}^{t-d_{1}} \dot{x}_{(s)} d s\right. \\
\left.\quad-3 \int_{t-d_{2}}^{t-d_{(t)}} \dot{x}_{(s)}^{T} d s E^{T} Q_{2} E \int_{t-d_{2}}^{t-d_{(t)}} \dot{x}_{(s)} d s\right) .
\end{array}
$$

By considering (5), it is obvious that

$$
\begin{gathered}
\left(x_{(t)}^{T} H_{1}+\dot{x}_{(t)}^{T} E^{T} H_{2}\right) \\
\times \sum_{i=0}^{m-1}\left(\left(A+A_{d}\right) x_{(t)}-m A_{d} \int_{t-((i+1) / m) d_{1}}^{t-(i / m) d_{1}} \dot{x}_{(s)} d s\right. \\
\left.-A_{d} \int_{t-d(t)}^{t-d_{1}} \dot{x}_{(s)} d s-E \dot{x}_{(t)}\right)=0 .
\end{gathered}
$$

Noting $E^{T} L=0$, we obtain

$$
\begin{aligned}
2 \sum_{i=0}^{m-1}\left(x_{(t)}^{T} S_{1}+x_{t-d(t)}^{T} S_{2}+x_{t-i \gamma}^{T} S_{3}\right. \\
+x_{t-(i+1) \gamma}^{T} S_{4}+\int_{t-(i+1) \gamma}^{t-i \gamma} \dot{x}_{(s)}^{T} d s S_{5} \\
\left.\quad+\int_{t-d_{t}}^{t-d_{1}} \dot{x}_{(s)}^{T} d s S_{6}+x_{t-d_{2}}^{T} S_{7}\right) L^{T} E \dot{x}_{(t)}=0 .
\end{aligned}
$$

Furthermore, according to the free-weighting-matrix method, we have

$$
\begin{aligned}
& \left(x_{(t)}^{T} G_{1}+x_{t-d(t)}^{T} G_{2}+x_{t-i \gamma}^{T} G_{3}+x_{t-(i+1) \gamma}^{T} G_{4}\right. \\
& +\dot{x}_{(t)}^{T} E^{T} G_{5}+\int_{t-(i+1) \gamma}^{t-i \gamma} \dot{x}_{(s)}^{T} d s G_{6} \\
& \left.+\int_{t-d_{t}}^{t-d_{1}} \dot{x}_{(s)}^{T} d s G_{7}+x_{t-d_{2}}^{T} G_{8}\right) \\
& \quad \times\left(x_{(t-i \gamma)}-x_{(t-(i+1) \gamma)}-\int_{t-(i+1) \gamma}^{t-i \gamma} \dot{x}_{(s)} d s\right)=0,
\end{aligned}
$$




$$
\begin{aligned}
& \left(x_{(t)}^{T} L_{1}+x_{t-d(t)}^{T} L_{2}+\dot{x}_{(t)}^{T} E^{T} L_{3}+\int_{t-d_{t}}^{t-d_{1}} \dot{x}_{(s)}^{T} d s L_{4}+x_{t-d_{2}}^{T} L_{5}\right) \\
& \times\left(x_{(t)}-x_{\left(t-d_{t}\right)}-\int_{t-d_{t}}^{t-d_{1}} \dot{x}_{(s)} d s-\sum_{i=0}^{m-1} \int_{t-(i+1) \gamma}^{t-i \gamma} \dot{x}_{(s)} d s\right)=0 .
\end{aligned}
$$

Then, combining manipulations (13)-(22) yields

$$
\dot{V}(t) \leq \sum_{i=0}^{m-1}\left(\theta(t) \xi_{(t)}^{T} \Psi_{1} \xi_{(t)}+(1-\theta(t)) \xi_{(t)}^{T} \Psi_{2} \xi_{(t)}\right)
$$

where

$$
\xi_{(t)}=\left[\begin{array}{lllllllll}
x_{(t)}^{T} & \int_{t-d_{(t)}}^{t-d_{1}} \dot{x}_{(s)}^{T} d s & x_{t-i \gamma}^{T} & x_{t-(i+1) \gamma}^{T} & \dot{x}_{(t)}^{T} E^{T} & \int_{t-(i+1) \gamma}^{t-i \gamma} & \dot{x}_{(s)}^{T} d s & x_{\left(t-d_{t}\right)}^{T} & x_{\left(t-d_{2}\right)}^{T}
\end{array}\right]^{T} .
$$

Then, we can obtain $\dot{V}(t)<0$ from (6). Thus, we can deduce that

$$
\dot{V}(t) \leq-\lambda_{2}\left\|\zeta_{(t)}\right\|^{2} \leq-\lambda_{2}\left\|x_{(t)}\right\|^{2}
$$

where $\lambda_{2}=-\max \left\{\lambda_{\max }\left(\Psi_{1}\right), \lambda_{\max }\left(\Psi_{2}\right)\right\}>0$. Therefore, the system (5) is stable based on Definition (2). This completes the proof.
Theorem 6. For the prescribed scalars satisfying $0<d_{1} \leq$ $d_{2}$, the singular system (1) is stabilizable for any time-delay $d_{(t)}$ satisfying $0<d_{1} \leq d_{(t)} \leq d_{2}$ if there exist positive symmetric matrices $P, U_{i}(i=1,2,3,4), Q_{1}, Q_{2}$ matrices $H_{1}=Z^{-1}\left[\begin{array}{ll}\bar{H}_{111} & 0 \\ \bar{H}_{121} & \bar{H}_{22}\end{array}\right] Z, H_{2}=\beta Z^{-1}\left[\begin{array}{cc}\beta^{-1} \bar{H}_{211} & 0 \\ \beta^{-1} \bar{H}_{221} & \bar{H}_{22}\end{array}\right] Z$, $S_{i}(i=1,2,3,4,5,6,7), G_{i}(i=1,2,3,4,5,6,7,8), L_{i}(i=$ $1,2,3,4,5), T \in R^{p \times n}$, and scalar $\beta$ satisfying the following LMIs:

$$
\widetilde{\Psi}_{i}=\left[\begin{array}{cccccccc}
\widetilde{\Psi}_{111} & \Psi_{112} & G_{1} & -G_{1} & \widetilde{\Psi}_{115} & \Psi_{116} & -L_{1}+L_{2}^{T} & L_{5}^{T} \\
* & \Psi_{i 22} & G_{7} & -G_{7} & \Psi_{125} & \Psi_{126} & -L_{2}^{T}-L_{4} & -L_{5}^{T} \\
* & * & \Psi_{133} & \Psi_{134} & \Psi_{135} & \Psi_{136} & G_{2}^{T} & \Psi_{138} \\
* & * & * & \Psi_{44} & \Psi_{145} & \Psi_{146} & -G_{2}^{T} & -G_{8}^{T} \\
* & * & * & * & \Psi_{155} & \Psi_{156} & L S_{2}^{T}-L_{3} & L S_{7}^{T} \\
* & * & * & * & * & \Psi_{166} & -G_{2}^{T}-L_{2}^{T} & \Psi_{168} \\
* & * & * & * & * & * & \Psi_{i 77} & \Psi_{i 78} \\
* & * & * & * & * & * & * & \Psi_{i 88}
\end{array}\right]<0, \quad i=1,2,
$$

where $L \in R^{n \times(n-r)}$ is any matrix with full columns and satisfies $E^{T} L=0, \bar{H}_{22} \in R^{p \times p}$ is any nonsingular matrix, $Z \in R^{n \times n}$ is any nonsingular constant matrix satisfying $Z B=\left[\begin{array}{ll}0 & B_{1}^{T}\end{array}\right]^{T}$, where $B_{1} \in R^{p \times p}$ is nonsingular, and $\widetilde{\Psi}_{111}=U_{1}+U_{2}+U_{3}+U_{4}+$ $\left\{L_{1}+H_{1}\left(A+A_{d}\right)+B T\right\}^{H}, \widetilde{\Psi}_{115}=\left(H_{2}\left(A+A_{d}\right)+\beta B T\right)^{T}+$ $E^{T} P-H_{1}+S_{1} L^{T}+L_{3}^{T}$. Then, a suitable state feedback controller is described as $K=\left(\bar{H}_{22} B_{1}\right)^{-1} B_{1} T$.

Proof. By replacing $A$ with $A+B K$ and choosing $K=$ $\left(\bar{H}_{22} B_{1}\right)^{-1} B_{1} T, H_{1}=Z^{-1}\left[\begin{array}{ll}\bar{H}_{111} & 0 \\ \bar{H}_{121} & \bar{H}_{22}\end{array}\right] Z$, and $H_{2}=$ $\beta Z^{-1}\left[\begin{array}{ll}\beta^{-1} \bar{H}_{211} & 0 \\ \beta^{-1} \bar{H}_{221} & \bar{H}_{22}\end{array}\right] Z$, it is easy to obtain $H_{1} B K=B T$ and $\mathrm{H}_{2} \mathrm{BK}=\beta B$ T. Thus, we can obtain (6) from LMIs (26). This completes the proof.

Theorem 7. For the prescribed scalars satisfying $0<d_{1} \leq d_{2}$, the singular system (1) is robustly stabilizable for any timedelay $d_{(t)}$ satisfying $0<d_{1} \leq d_{(t)} \leq d_{2}$ if there exist positive symmetric matrices $P, U_{i}(i=1,2,3,4), Q_{1}, Q_{2}$, matrices $H_{1}=Z^{-1}\left[\begin{array}{cc}\bar{H}_{111} & 0 \\ \bar{H}_{121} & \bar{H}_{22}\end{array}\right] Z, H_{2}=\beta Z^{-1}\left[\begin{array}{cc}\beta^{-1} \bar{H}_{211} & 0 \\ \beta^{-1} \bar{H}_{221} & \bar{H}_{22}\end{array}\right] Z$,
$S_{i}(i=1,2,3,4,5,6,7), G_{i}(i=1,2,3,4,5,6,7,8), L_{i}(i=$ $1,2,3,4,5), T \in R^{p \times n}$, and scalars $\beta, \lambda>0$ satisfying the following LMIs:

$$
\left[\begin{array}{ccc}
\widetilde{\Psi}_{i} & \widetilde{\Pi}_{2}^{T} & \lambda \widetilde{\Lambda}_{2}^{T} \\
* & -\lambda I & 0 \\
* & * & -\lambda I
\end{array}\right]<0, \quad i=1,2
$$

where $\widetilde{\Psi}_{1}, \widetilde{\Psi}_{2}$, and L follow the same definitions as in Theorem 6 and $\bar{H}_{22} \in R^{p \times p}$ is any nonsingular matrix, $Z \in R^{n \times n}$ is any nonsingular constant matrix satisfying $Z B=$ $\left[\begin{array}{ll}0 & B_{1}^{T}\end{array}\right]^{T}$, where $B_{1} \in R^{p \times p}$ is nonsingular, and $\widetilde{\Pi}_{2}=\left[\begin{array}{llllllllll}M^{T} & H_{1}^{T} & 0 & 0 & 0 & M^{T} H_{2}^{T} & 0 & 0 & 0\end{array}\right], \widetilde{\Lambda}_{2}=$ $\left[\begin{array}{lllllll}N_{1}+N_{2} & -N_{2} & 0 & 0 & 0-m N_{2} & 0 & 0\end{array}\right]$. Then, a suitable state feedback control law is described as $K=\left(\bar{H}_{22} B_{1}\right)^{-1} B_{1} T$.

Proof. Replacing $A$ and $A_{d}$ with $A+\Delta A$ and $A_{d}+\Delta A_{d}$, respectively, (26) can be expressed as

$$
\widetilde{\Psi}_{i}+\widetilde{\Lambda}_{2}^{T} F_{(t)} \widetilde{\Pi}_{2}+\widetilde{\Pi}_{2}^{T} F_{(t)}^{T} \widetilde{\Lambda}_{2}<0, \quad i=1,2
$$


TABLE 1: Comparisons of the upper bounds of $d_{2}$ with different $d_{1}$ of the system without uncertainties.

\begin{tabular}{lcccc}
\hline$d_{1}$ & 0.5 & 1 & 1.5 & 2 \\
\hline$[32]$ & 2.9056 & 2.9073 & 2.9103 & 2.9163 \\
{$[33]$} & 3.0401 & 3.0416 & 3.0441 & 3.0490 \\
{$[34]$} & 3.0401 & 3.0416 & 3.0441 & 3.0490 \\
Theorem 5 & 3.0501 & 3.0540 & 3.0604 & 3.0724 \\
\hline
\end{tabular}

By Lemma 4, LMIs (28) hold for any $F_{(t)}$ satisfying $F_{(t)}^{T} F_{(t)} \leq I$ if and only if there exists scalar $\lambda>0$ such that

$$
\widetilde{\Psi}_{i}+\lambda \widetilde{\Lambda}_{2}^{T} \widetilde{\Lambda}_{2}+\lambda^{-1} \widetilde{\Pi}_{2}^{T} \widetilde{\Pi}_{2}<0, \quad i=1,2 .
$$

Applying the Schur complement, (27) is equivalent to (29). This completes the proof.

\section{Illustrative Examples}

Example 1. Consider that the continuous singular timevarying delay system (5) has the system matrices of $E=\left[\begin{array}{ll}9 & 3 \\ 6 & 2\end{array}\right]$, $A=\left[\begin{array}{ll}-13.1 & -13.7 \\ -15.4 & -23.8\end{array}\right]$, and $A_{d}=\left[\begin{array}{ll}-18.6 & -10.4 \\ -25.2 & -16.8\end{array}\right]$. Set $\mu=0.2$ and select $L=\left[\begin{array}{ll}2 & -3\end{array}\right]^{T}$. For the deferent lower bounds $d_{1}$, the upper bounds of $d_{2}$ for the system to be admissible are shown in Table 1. It is obvious that the delay-dependent stability results obtained in this paper are better than those in [32-34].

Example 2. Consider that the continuous singular timevarying delay system has the following system matrices [25]:

$$
\begin{gathered}
E=\left[\begin{array}{ccc}
1 & 1 & 0 \\
1 & -1 & 1 \\
2 & 0 & 1
\end{array}\right], \quad A=\left[\begin{array}{ccc}
2 & 1 & 1 \\
-1 & 0 & 1 \\
0.5 & 0 & 1
\end{array}\right], \\
A_{d}=\left[\begin{array}{ccc}
-1.5 & 0.5 & -0.8 \\
1 & 1 & 0.5 \\
0.7 & 0.5 & 1
\end{array}\right], \quad B=\left[\begin{array}{cc}
1 & 2 \\
1.5 & 0 \\
0 & 1
\end{array}\right] .
\end{gathered}
$$

Set $\mu=0$ and select $L=\left[\begin{array}{lll}-1 & 1 & 2\end{array}\right]^{T}$; Theorem 5 yields that the system is stable for any constant delay $d$ satisfying $0 \leq d \leq 5$, which has less conservatism than $0 \leq d \leq 3.1$ which was obtained in [28]. Then, set $\mu=0.2$ and select $L=\left[\begin{array}{lll}-1 & 1 & 2\end{array}\right]^{T}$. For any time-varying delay $d_{(t)}$ satisfying $0.5 \leq d_{(t)} \leq 2$, the LMIs (26) are feasible, and a controller gain law can be obtained as follows:

$$
K=\left[\begin{array}{ccc}
-0.6466 & -0.1275 & -0.2681 \\
0.2072 & 0.5078 & -0.1450
\end{array}\right] .
$$

Now, we consider the system uncertainties, and the uncertain system matrices have the following forms of $M=\left[\begin{array}{lll}0.4 & 0.3 & 0.1\end{array}\right]^{T}, N_{1}=\left[\begin{array}{lll}0.2 & 0.4 & 0.5\end{array}\right]$, and $N_{2}=$ $\left[\begin{array}{lll}0.3 & 0.7 & 0.5\end{array}\right]$. Set $\mu=0.2$ and choose $L=\left[\begin{array}{lll}-1 & 1 & 2\end{array}\right]^{T}$. For any time-varying delay $d_{(t)}$ satisfying $0.5 \leq d_{(t)} \leq 2$, the LMIs (27) are feasible, and a robust controller can be obtained as

$$
K=\left[\begin{array}{ccc}
-2.2790 & -0.4928 & -0.9442 \\
0.7654 & 1.6355 & -0.4224
\end{array}\right] \text {. }
$$

\section{Conclusion}

In this research, the robustly delay-dependent stabilization for continuously singular time-varying delay systems with norm-bounded uncertainties is investigated. Based on Lyapunov stability theory and LMI technique, the new delaydependent LMIs-based conditions are established for the singular time-varying delay system to be regular, impulse free, and stable. By solving these LMIs, the desired state feedback control law can be obtained, and the regularity, causality, and stability of the closed-loop system are guaranteed. Finally, simulation results are given to show the effectiveness of the proposed method.

\section{Conflict of Interests}

The authors declare that there is no conflict of interests regarding the publication of this paper.

\section{Acknowledgments}

This work was partially supported by the National Natural Science Foundation (nos. 61305019 and 51365017), Jiangxi Provincial Natural Science Foundations (nos. GJJ13430 and GJJ13385), and the Natural Science Foundation of Jiangxi University of Science and Technology (no. NSFJ2014-K16) of China.

\section{References}

[1] F. Weng, L. Liang, Z. Yu, and Y. Ding, "Online parameterdependent controller design for electro-hydraulic servo systems driven by double-rod actuators," Journal of Computational Information Systems, vol. 8, no. 22, pp. 9507-9514, 2012.

[2] F. Weng, Y. Ding, L. Liang, and G. Yang, "Fault tolerant vibration-attenuation controller design for uncertain linear structural systems with input time-delay and saturation," Shock and Vibration, vol. 20, no. 5, pp. 963-977, 2013.

[3] R. Lu, B. Lou, and A. Xue, "Mode-dependent quantised filtering for Markovian jump singular system," International Journal of Systems Science, 2013.

[4] J. Wei and R. Ma, "Stability and stabilization of continuous-time Markovian jump singular systems with partly known transition probabilities," Mathematical Problems in Engineering, vol. 2014, Article ID 752626, 8 pages, 2014.

[5] Q. Fang, J. Peng, and F. Cao, "Synchronization and control of linearly coupled singular systems," Mathematical Problems in Engineering, vol. 2013, Article ID 230741, 10 pages, 2013.

[6] J. Lin and L. Rong, "Robust stability and stabilization for singular time-delay systems with linear fractional uncertainties: a strict LMI approach," Mathematical Problems in Engineering, vol. 2013, Article ID 598357, 11 pages, 2013.

[7] F. Weng and W. Mao, "Analysis of robust stability for uncertain discrete singular systems with time-varying delay," Journal of Southeast University, vol. 42, no. 1, pp. 92-97, 2012.

[8] R. Lu, H. Su, J. Chu, and A. Xue, "A simple approach to robust d-stability analysis for uncertain singular delay systems," Asian Journal of Control, vol. 11, no. 4, pp. 411-419, 2009. 
[9] R. Lu, H. Su, J. Chu, S. Zhou, and M. Fu, "Reduced-order $H_{\infty}$ filtering for discrete-time singular systems with lossy measurements," IET Control Theory and Applications, vol. 4, no. 1, pp. 151-163, 2010.

[10] R. Lu, H. Li, and Y. Zhu, "Quantized $H_{\infty}$ filtering for singular time-varying delay systems with unreliable communication channel," Circuits, Systems, and Signal Processing, vol. 31, no. 2, pp. 521-538, 2012.

[11] Y. Ding, F. Weng, and W. Mao, "Improved stability and stabilization criteria of singular system with time-delay and parameter uncertainties," Journal of Information \& Computational Science, vol. 10, no. 18, pp. 6067-6076, 2013.

[12] R. Lu, H. Wu, and J. Bai, "New delay-dependent robust stability criteria for uncertain neutral systems with mixed delays," Journal of the Franklin Institute, vol. 351, no. 3, pp. 1386-1399, 2014.

[13] Z. Wu, P. Shi, H. Su, and J. Chu, "Stochastic synchronization of Markovian jump neural networks with time-varying delay using sampled-data," IEEE Transactions on Cybernetics, vol. 43, no. 6, pp. 1796-1806, 2013.

[14] Z.-G. Wu, P. Shi, H. Su, and J. Chu, "Passivity analysis for discrete-time stochastic markovian jump neural networks with mixed time delays," IEEE Transactions on Neural Networks, vol. 22, no. 10, pp. 1566-1575, 2011.

[15] Z.-G. Wu, P. Shi, H. Su, and J. Chu, "Sampled-data exponential synchronization of complex dynamical networks with timevarying coupling delay," IEEE Transactions on Neural Networks and Learning Systems, vol. 24, no. 8, pp. 1177-1187, 2013.

[16] X. Ji, Z. Yang, and H. Su, "Robust stabilization for uncertain discrete singular time-delay systems," Asian Journal of Control, vol. 12, no. 2, pp. 216-222, 2010.

[17] X. Sun, Q. Zhang, C. Yang, and Z. Su, "Stability analysis and stabilization for discrete-time singular delay systems," Journal of Systems Engineering and Electronics, vol. 22, no. 3, pp. 482487, 2011.

[18] X. Ji, C. Zu, and H. Su, "Delay-dependent synchronisation for singular Lur'e systems using time delay feedback control," International Journal of Modelling, Identification and Control, vol. 19, no. 2, pp. 125-133, 2013.

[19] Y. C. Ding, F. L. Weng, and Z. A. Yu, "Actuator saturation and control design for buildings structural systems with improved uncertainty description," Shock and Vibration, vol. 20, no. 2, pp. 297-308, 2013.

[20] S. Zhu, Z. Cheng, and J. Feng, "Delay-dependent robust stability criterion and robust stabilization for uncertain singular timedelay systems," in Proceedings of the American Control Conference (ACC '05), pp. 2839-2844, June 2005.

[21] S. Zhu, C. Zhang, Z. Cheng, and J. Feng, "Delay-dependent robust stability criteria for two classes of uncertain singular time-delay systems," IEEE Transactions on Automatic Control, vol. 52, no. 5, pp. 880-885, 2007.

[22] J. Gao, M. Lei, and H. Pan, "New delay-dependent criteria for stability and stabilization of uncertain singular systems with time delay," in Proceedings of the IEEE International Conference on Automation and Logistics (ICAL '09), pp. 703-708, August 2009.

[23] R. Yang, H. Gao, and P. Shi, "Delay-dependent robust control for uncertain stochastic time-delay systems," International Journal of Robust and Nonlinear Control, vol. 20, pp. 1852-1865, 2010.
[24] F. Weng and W. Mao, "Robust stability and stabilization of uncertain discrete singular time-delay systems based on PNP Lyapunov functional," IMA Journal of Mathematical Control and Information, vol. 30, no. 3, pp. 301-314, 2013.

[25] P. He, C. Jing, T. Fan, and C. Chen, "Robust adaptive synchronization of complex networks with multiple coupling time-varying delays," International Journal of Automation and Control, vol. 7, no. 4, pp. 223-248, 2013.

[26] F. Weng, Y. Ding, G. Yang, L. Liang, and Z. Yu, "Less conservative stability criteria for discrete-time nonlinear stochastic singular systems with mixed time-delay," Asian Journal of Control, vol. 15, no. 6, pp. 1871-1876, 2013.

[27] F. Weng and W. Mao, "Delay-range-dependent and delaydistribution-independent stability criteria for discrete-time singular Markovian jump systems," International Journal of Control, Automation and Systems, vol. 11, no. 2, pp. 233-242, 2013.

[28] Z.-G. Wu and W.-N. Zhou, "Delay-dependent robust stabilization for uncertain singular systems with state delay," Acta Automatica Sinica, vol. 33, no. 7, pp. 714-718, 2007.

[29] L. Dai, Singular Control Systems, Springer, Berlin, Germany, 1989.

[30] Y. Ding, F. Weng, J. Ge, L. Liang, and G. Yang, "Finite-time vibration-attenuation controller design for structural systems with parameter uncertainties," Advances in Mechanical Engineering, vol. 2014, Article ID 673174, 11 pages, 2014.

[31] Y. Ding, F. Weng, and L. Liang, "Active vibration attenuation for uncertain buildings structural systems with sensor faults," Journal of Computers, vol. 8, no. 12, pp. 3072-3078, 2013.

[32] H. Hu, Q. Zhang, and D. Zhao, "Delay-dependent stability of uncertain descriptor system with interval time-varying delay," International Journal of Information and Systems Sciences, vol. 4, no. 4, pp. 534-547, 2008.

[33] A. Haidar and E. K. Boukas, "Exponential stability of singular systems with multiple time-varying delays," Automatica, vol. 45, no. 2, pp. 539-545, 2009.

[34] Z. Wu, H. Su, and J. Chu, " $H_{\infty}$ filtering for singular systems with time-varying delay," International Journal of Robust and Nonlinear Control, vol. 20, no. 11, pp. 1269-1284, 2010. 


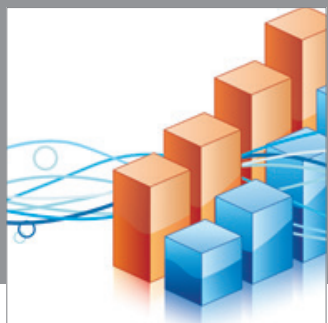

Advances in

Operations Research

mansans

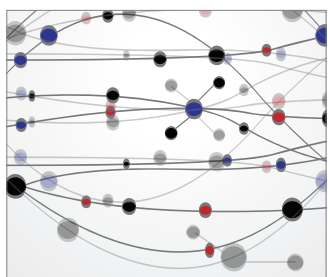

The Scientific World Journal
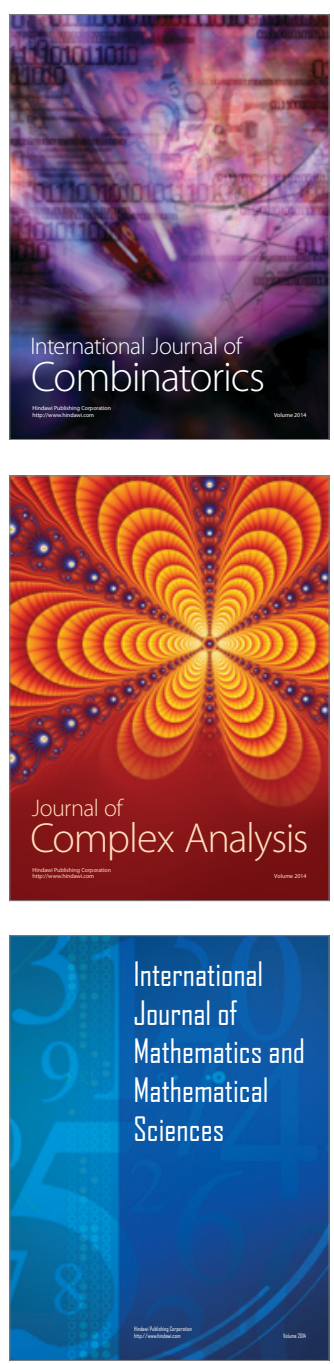
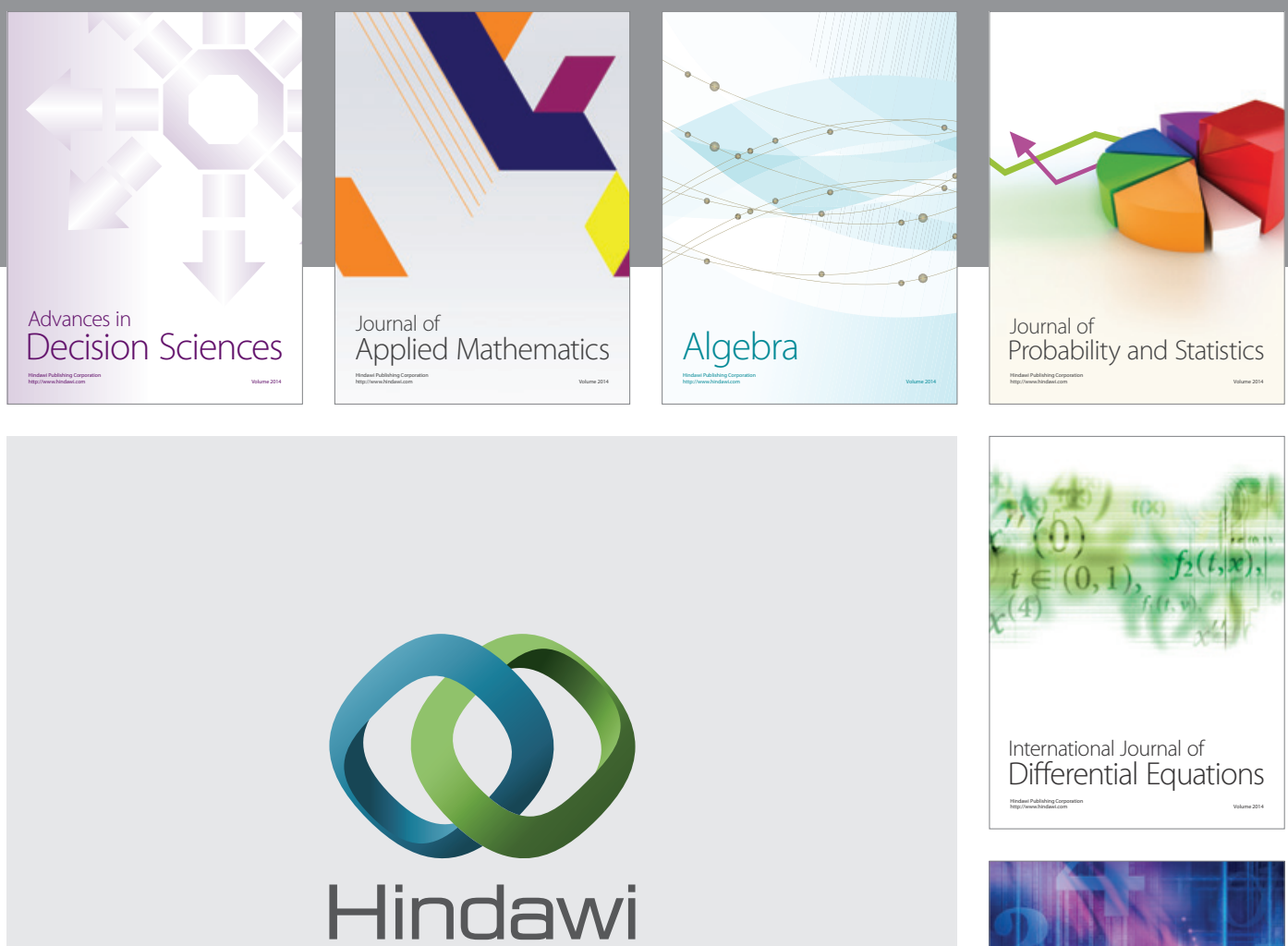

Submit your manuscripts at http://www.hindawi.com
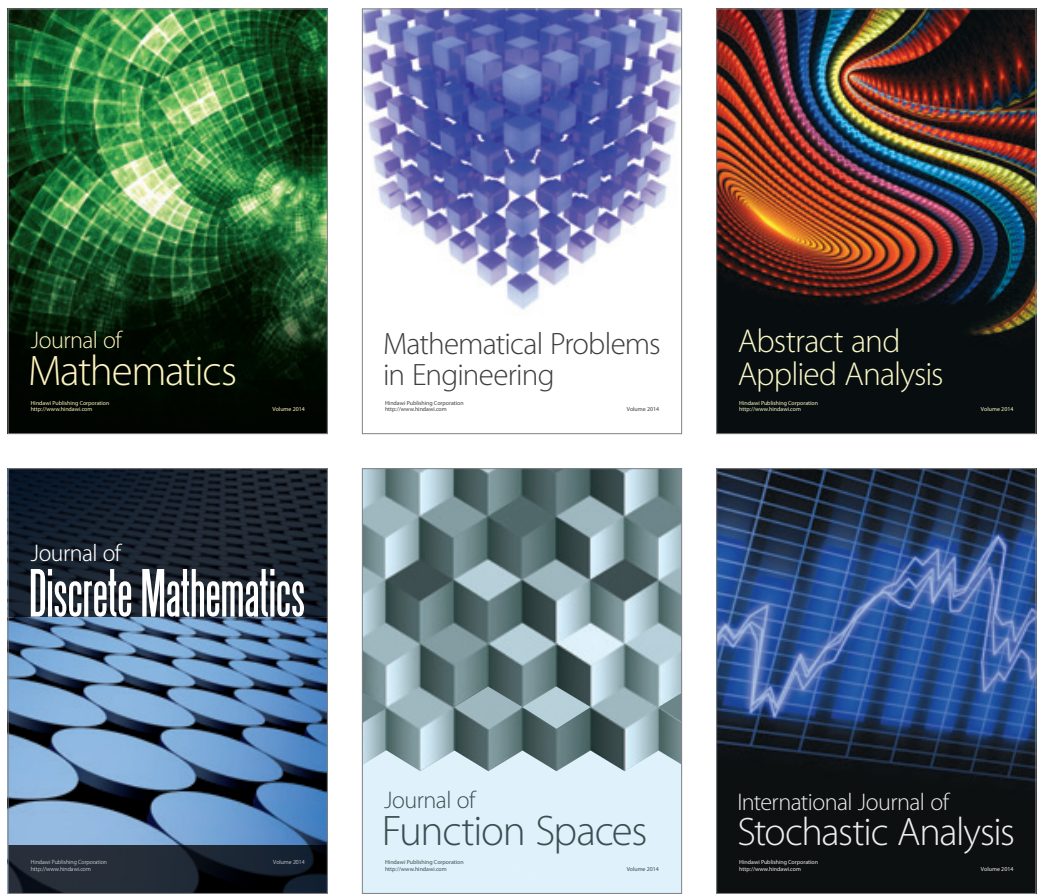

Journal of

Function Spaces

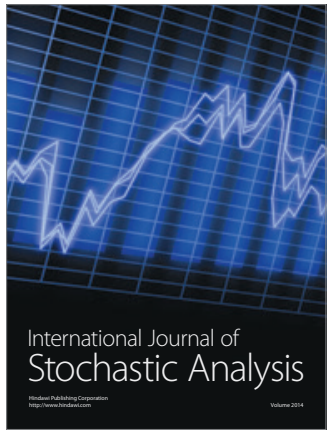

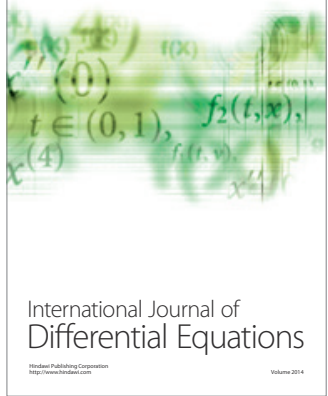
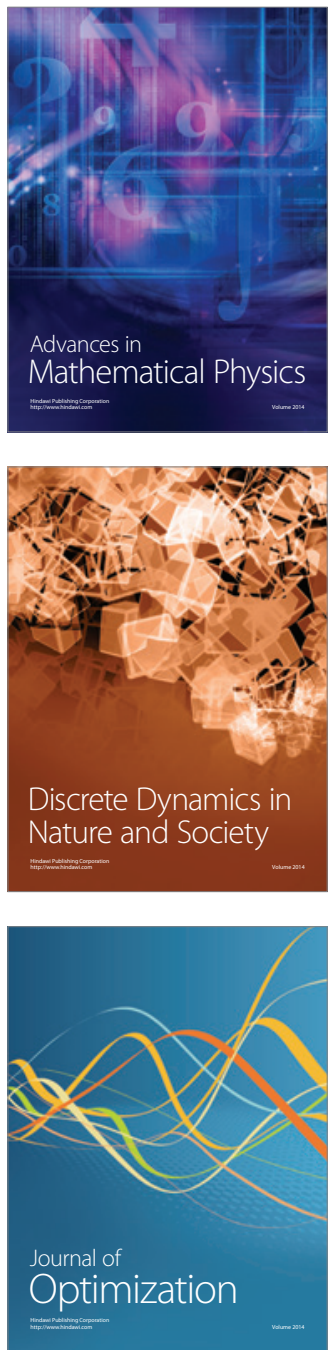